\title{
Stroke and atrial fibrillation: is stroke prevention treatment appropriate beforehand?
}

D Deplanque, F Corea, C Arquizan, L Parnetti, J L Mas, V Gallai, D Leys, and the SAFE I Study Investigators

\begin{abstract}
Objective-To undertake a pilot study before conducting a large European multicentre prospective study, to determine the proportion of patients with atrial fibrillation who were not receiving antithrombotic treatment before stroke onset, and their characteristics.

Design and patients-The stroke in atrial fibrillation ensemble (SAFE) I study was an observational study conducted in 213 patients with atrial fibrillation consecutively admitted in 1997 to three European centres for an acute stroke or transient ischaemic attack (TIA). It was determined whether they were receiving prior antithrombotic treatment.
\end{abstract}

Results-Atrial fibrillation was known before stroke in 148 patients $(69.5 \%)$. Of 213 patients, $34(16.0 \%)$ were receiving anticoagulation treatment before stroke, but only six had an international normalised ratio between 2.0 and $3.5 ; 65(30.5 \%)$ were receiving antiplatelet treatment; and three $(1.4 \%)$ were receiving both anticoagulation and antiplatelet treatment. Of 137 patients eligible for oral anticoagulation, $108(78.8 \%)$ did not receive treatment. Of 142 patients eligible for any antithrombotic treatment, $62 \quad(43.7 \%)$ were not treated. The logistic regression analysis, assuming anticoagulation treatment as a dependent variable, found digoxin treatment, absence of arterial hypertension, mitral stenosis, and cardioversion as independent factors. Assuming any antithrombotic treatment as a dependent variable, previously known atrial fibrillation, lower age, being a nonsmoker, and absence of arterial hypertension were found to be independent factors.

Conclusion-More than half of the patients with atrial fibrillation admitted for acute stroke or TIA were not receiving any antithrombotic treatment beforehand. New onset atrial fibrillation and contraindications account for a minority of nonprescriptions; thus, other reasons should be identified to improve stroke prevention in the community.

(Heart 1999;82:563-569)

Keywords: stroke; atrial fibrillation; anticoagulant treatment; antiplatelet treatment

Non-valvar atrial fibrillation (NVAF) increases the risk of stroke fivefold ${ }^{12}$ and accounts for nearly $25 \%$ of strokes occurring in people over 80 years old. ${ }^{2}$ Moreover, NVAF leads to more severe strokes than other causes, ${ }^{3}$ with a combined rate of death or significant neurological disability of $71 \%$ one year after stroke onset. ${ }^{4}$ The risk of stroke in NVAF is particularly increased in subjects with arterial hypertension, recent congestive heart failure, prior transient ischaemic attacks (TIAs) or stroke, or echocardiographic evidence of left ventricular dysfunction, mitral annular calcification, or left atrial enlargement. ${ }^{5}{ }^{6}$ Prevention is critical to reduce death and disability in patients with NVAF, because the annual rate of recurrent stroke of any severity is $12 \%$ in the absence of treatment. ${ }^{7}$

In both primary and secondary stroke prevention in NVAF patients, it has been shown that the risk of stroke was significantly reduced by warfarin and to a lesser extent by aspirin. ${ }^{89}$ The results of these trials were so impressive that most of them were stopped prematurely. ${ }^{10}$ However, prescription of such a treatment should take into account high risk patients as defined in the third stroke prevention in atrial fibrillation (SPAF III) study. ${ }^{811}$

Despite these results, day to day practice seems to differ. Indeed, physician surveys suggest that not all appropriate patients with NVAF receive anticoagulation treatment. ${ }^{12-22}$ In a community study conducted in 1994 in the USA, $62 \%$ of patients with known NVAF and no contraindications to anticoagulation did not receive anticoagulation, two thirds of them did not receive aspirin, and the degree of anticoagulation treatment was appropriate in only $60 \%$ of treated patients. ${ }^{15}$ In an Australian centre, of 103 patients with chronic NVAF who were consecutively admitted for various reasons, including 12 strokes and two TIAs, two thirds would have been ideal candidates, but less than $10 \%$ were receiving anticoagulation treatment. ${ }^{18}$

It is not possible to draw general conclusions from these studies because the health systems differ from country to country, the patterns of anticoagulation differ, ${ }^{23}$ most of these studies were conducted before the results of important trials, and previous information campaigns may increase the proportion of patients treated with anticoagulants or aspirin for atrial fibrillation. ${ }^{24}$

Moreover, little is known about the current use of anticoagulation treatment in patients with atrial fibrillation in continental Europe. Determining the proportion of stroke patients with atrial fibrillation who did not receive appropriate treatment, and the reasons, is 
Table 1 Description of the study population

\begin{tabular}{|c|c|c|c|}
\hline Number of patients & 213 & \multicolumn{2}{|c|}{ Past (but interrupted) treatments ( $(\%)$ ) } \\
\hline Perugia & $38(17.8 \%)$ & Oral anticoagulation & $12(5.6)$ \\
\hline Paris & $32(15.0 \%)$ & Mean delay since interruption & 1.00 year $(0-7)$ \\
\hline Lille & $143(67.1 \%)$ & \multicolumn{2}{|c|}{$\begin{array}{l}\text { Reasons: anaemia (1), surgery (1), frequent falls (1), poor } \\
\text { observance (2), reduction of atrial fibrillation ( } 2)\end{array}$} \\
\hline Demography & & Aspirin treatment & $1(0.5)$ \\
\hline Mean age at stroke onset & 77.63 years (range $34-96$ ) & Cardioversion & $8(3.8)$ \\
\hline \multirow[t]{2}{*}{ Male sex } & $98(46 \%)$ & Electrical & $2(0.9)$ \\
\hline & & Pharmacological & $8(3.8)$ \\
\hline Atrial fibrillation ( $n(\%)$ ) & & Both & $1(0.5)$ \\
\hline Previously known & $147(69.5)$ & & \\
\hline With mitral stenosis & $8(3.8)$ & \multicolumn{2}{|c|}{ Current treatments just before stroke (n (\%)) } \\
\hline With thyrotoxicosis & $3(1.4)$ & Oral anticoagulation & $34(16.0)$ \\
\hline \multirow[t]{2}{*}{ Thrombus on echography } & $3(1.4)$ & INR between $2-3.5$ & $6(2.8)$ \\
\hline & & Aspirin treatment & $62(29.1)$ \\
\hline Index stroke (n(\%)) & & Other antiplatelet drugs & $6(2.8)$ \\
\hline Ischaemic stroke & $151(70.9)$ & Any antithrombotic ${ }^{\star}$ & $99(40.7)$ \\
\hline Haemorrhagic stroke & $18(8.5)$ & Digoxin & $84(39.4)$ \\
\hline Haemorrhagic infarct & $17(8.0)$ & $\beta$ blockers & $38(17.8)$ \\
\hline \multirow[t]{2}{*}{ TIA } & $27(12.7)$ & Calcium channel blockers & $53(24.9)$ \\
\hline & & ACE inhibitors & $56(26.3)$ \\
\hline Medical history (n (\%)) & & Diuretics & $80(37.6)$ \\
\hline Previous stroke or TIA & $51(23.9)$ & Nitrates & $49(23.0)$ \\
\hline Previous cerebral haemorrhage & $1(0.5)$ & Insulin & $7(3.3)$ \\
\hline Previous systemic emboli & $5(2.4)$ & Antiarrhythmics & $53(24.9)$ \\
\hline Non-life threatening bleedings & $2(0.9)$ & Oral hypoglycaemic drugs & $14(6.6)$ \\
\hline Life threatening bleedings & $0(0.0)$ & Hormonal treatment (in women) & $2(1.7)$ \\
\hline Depression & $14(6.6)$ & Antidepressant & $10(4.7)$ \\
\hline Dementia & $12(5.6)$ & Other cerebroactive drugs & $10(4.7)$ \\
\hline Angina pectoris & $58(27.2)$ & & \\
\hline Congestive heart failure & $32(15.0)$ & Treatment at discharge $(n(\%))+$ & \\
\hline Active gastroduodenal ulcer & $6(2.8)$ & Oral anticoagulation & $74(41.6)$ \\
\hline Peripheral artery disease & $16(7.5)$ & Aspirin & $73(41.0)$ \\
\hline Alcoholism & $11(5.2)$ & Other antiplatelet drugs & $7(3.9)$ \\
\hline Headache & $3(1.4)$ & Any antithrombotic & $154(86.5)$ \\
\hline \multirow[t]{2}{*}{ Allergy to aspirin } & $0(0.0)$ & Digoxin & $66(37.1)$ \\
\hline & & $\beta$ blockers & $27(15.2)$ \\
\hline \multicolumn{2}{|c|}{ Risk factors for strokes and other potential causes ( $(\%))$} & Calcium channel blockers & $37(20.8)$ \\
\hline Arterial hypertension & $152(71.4)$ & ACE inhibitors & $44(24.7)$ \\
\hline Diabetes mellitus & $30(14.1)$ & Diuretics & $55(30.9)$ \\
\hline Hyperlipidaemia & $54(25.4)$ & Nitrates & 39 (21.9) \\
\hline Abnormal haematocrit & $30(14.1)$ & Insulin & $12(6.7)$ \\
\hline Smoking & $24(11.3)$ & Antiarrhythmics & $46(25.8)$ \\
\hline Other high risk cardiopathies & $14(6.6)$ & Oral hypoglycaemic drugs & $11(6.2)$ \\
\hline Stenosis of the ICA $\geqslant 50 \%$ & $28(13.2)$ & Hormonal treatment & $2(1.1)$ \\
\hline \multirow[t]{2}{*}{ Familial history of stroke } & $25(11.7)$ & Antidepressants & $9(5.1)$ \\
\hline & & Other cerebroactive drugs & $21(11.8)$ \\
\hline \multicolumn{4}{|l|}{$C T$ brain scan (n (\%)) } \\
\hline Multiple infarcts & $47(22.1)$ & In-hospital death (n (\%)) & $35(16.4)$ \\
\hline White matter changes & $96(45.1)$ & & \\
\hline
\end{tabular}

The total may differ from $100 \%$ because of rounding.

*Three patients received anticoagulation and antithrombotic drugs simultaneously.

+Percentages were calculated in survivors at discharge.

ACE, angiotensin converting enzyme; ICA, internal carotid artery; INR, international normalised ratio; TIA, transient ischaemic attack.

necessary to focus future strategies to improve stroke prevention in the community. To address this question, a large prospective multicentre and multinational study is required. Before starting such a study, a pilot study is necessary.

The objective of the stroke in atrial fibrillation ensemble (SAFE) I study was to determine the proportion of patients with atrial fibrillation admitted for acute stroke who were not receiving antithrombotic treatment before stroke and to compare their characteristics with those of patients who did. The reasons why physicians did not prescribe any antithrombotic drug were not evaluated in this study.

\section{Patients and methods}

PATIENTS

SAFE I was an observational study conducted in 213 consecutive patients admitted to three European university centres (Perugia, Italy; Paris Sainte-Anne Hospital, France; Lille R Salengro Hospital, France), between 1 January and 31 December 1997. All patients, of any age and sex, admitted to the centres during the study period were eligible to enter the study, provided they were admitted for an acute stroke (ischaemic or haemorrhagic) or TIA, and had atrial fibrillation either on the admission ECG or on a previous occasion. The characteristics of the study population are given in table 1 .

\section{METHODS}

At admission all patients underwent noncontrast computed tomography (CT), routine biological tests, 12 lead ECG, and chest radiography. The international normalised ratio (INR) values were determined at admission. Patients with ischaemic stroke or TIA underwent cervical Doppler ultrasonography. All patients with an ischaemic stroke or TIA, and most patients with haemorrhagic stroke, underwent a transthoracic echocardiography. Magnetic resonance angiography, magnetic resonance imaging, conventional angiography, and transoesophageal echocardiography, were performed in selected patients. Ischaemic stroke was defined as clinical signs of focal disturbance of cerebral function lasting longer than 24 hours or leading to death, with no apparent cause other than of vascular origin, 
and no sign of relevant primary intracerebral haemorrhage on CT scan or at necropsy. Haemorrhagic stroke was defined as clinical signs of focal disturbance of cerebral function lasting more than 24 hours or leading to death, with no apparent cause other than vascular, and evidence of a relevant primary intracerebral haemorrhage on CT scan or at necropsy. Patients with haemorrhagic changes within the infarct were classified in the haemorrhagic infarct group. TIAs were defined as episodes of focal cerebral dysfunction, presumably ischaemic in origin, lasting less than 24 hours and followed by return to normality, without any relevant lesion on CT scan other than of ischaemic origin. Medical history was determined from all available records (letter from their general practitioner (GP) or telephone call) and sources (patient, family, or GP).

We collected the following data: age; sex; previous stroke or TIA; presence of arterial hypertension (defined as systolic blood pressure $\geqslant 160 \mathrm{~mm} \mathrm{Hg}$, or diastolic blood pressure $\geqslant 90 \mathrm{~mm} \mathrm{Hg}$, or treatment with antihypertensive drugs before stroke onset); diabetes mellitus (defined as fasting serum glucose concentration $\geqslant 120 \mathrm{mg} / \mathrm{dl}(6.7 \mathrm{mmol} / \mathrm{l})$, or current use of antidiabetic drugs); hyperlipidaemia (defined as fasting serum triglycerides concentration $\geqslant 150 \mathrm{mg} / \mathrm{dl}(1.71 \mathrm{mmol} / \mathrm{l})$, or fasting cholesterol serum concentration $\geqslant 230 \mathrm{mg} / \mathrm{dl}$ $(6.0 \mathrm{mmol} / \mathrm{l})$, or current hypolipidaemic treatment); history of peripheral artery disease with intermittent claudication; alcoholism (defined as a mean alcohol consumption $\geqslant 300 \mathrm{~g} /$ week); cigarette smoking ( $\geqslant 10$ cigarettes/day or cessation less than five years earlier); high risk cardiopathies as defined according to the TOAST (trial of org 10172 in acute stroke treatment) criteria ${ }^{25}$; and significant stenosis of the internal carotid arteries defined as $\geqslant 50 \%$ narrowing of the lumen documented by Doppler ultrasonography, B mode echotomography, magnetic resonance, or conventional angiography. Treatments at entrance and at discharge were also recorded.

STATISTICAL ANALYSES

The first step of the statistical analysis determined the percentage of subjects who were receiving oral anticoagulation treatment, antiplatelet treatment, both, or neither. In patients receiving anticoagulation treatment, those whose INR value at admission was between 2.0-3.5 were considered as being properly treated, those with an INR value $<2$ were considered as undertreated, and those with an INR value $>3.5$ were considered as overtreated.

The second step comprised a bivariate analysis comparing variables between patients who were receiving oral anticoagulation treatment before stroke and patients who were not. We used the $\chi^{2}$ test with Yates correction or Fisher's exact test when appropriate and the odds ratio (OR) method with $95 \%$ confidence interval $(\mathrm{CI})$ to compare qualitative factors between groups; the unpaired $t$ test was used to compare quantitative variables. Variables were demographic details, length of time of atrial fibrillation, characteristics of the index stroke, medical history, risk factors for stroke, potential causes of stroke other than atrial fibrillation, CT findings, and past or current treatments. The same analysis was also performed between patients who were under any antithrombotic treatment (oral anticoagulants or antiplatelet agents) before stroke and patients who were not.

The third step comprised a logistic regression analysis assuming anticoagulation before stroke (quoted 1 when present and 0 when absent) as dependent variable, the independent variables included in the analysis being selected from the bivariate analysis with a 0.25 level as a screening criterion. ${ }^{26}$ Another logistic regression analysis was performed assuming any antithrombotic treatment (oral anticoagulants, antiplatelet agents, or both) before stroke (quoted 1 when present and 0 when absent) as dependent variable. Colinearity between variables (defined as $r>0.6$ ) was excluded.

Patients were regarded as eligible for oral anticoagulation treatment when they had known atrial fibrillation, no recent systemic or cerebral bleeding, no alcoholism, no history of falls, and no known cognitive dysfunction leading to difficulties to manage anticoagulant treatment. Patients were regarded as eligible for antiplatelet treatment when they had known atrial fibrillation, no past gastrointestinal bleeding, no recent gastrointestinal ulcers, and no allergy to antiplatelet drugs. Age was not regarded as a contraindication for anticoagulation treatment because there was no upper age limit in the inclusion criteria of most trials conducted in patients with NVAF. Data were analysed using the SPSS/Macintosh package.

Table 2 Comparison between patients with and without anticoagulation treatment at admission

\begin{tabular}{|c|c|c|c|c|c|}
\hline & \multicolumn{2}{|c|}{ Oral anticoagulation } & \multirow[b]{2}{*}{ Odds ratio } & \multirow{2}{*}{$\begin{array}{l}95 \% \text { confidence } \\
\text { intervals }\end{array}$} & \multirow[b]{2}{*}{$p$ Value } \\
\hline & Yes $(n=34)$ & No $(n=179)$ & & & \\
\hline Age $(\text { years })^{\star}$ & 74.9 & 78.1 & & & 0.0645 \\
\hline Atrial fibrillation previously known & $30(88.2)$ & $118(65.9)$ & 3.88 & 1.30 to 11.55 & 0.0096 \\
\hline Mitral stenosis & $4(11.8)$ & $4(2.2)$ & 5.83 & 1.38 to 24.72 & 0.0239 \\
\hline Ischaemic stroke & $17(50.0)$ & $134(74.9)$ & 0.34 & 0.16 to 0.71 & 0.0034 \\
\hline Haemorrhagic stroke & $12(35.3)$ & $6(3.4)$ & 15.73 & 5.34 to 46.29 & 0.0001 \\
\hline Previous stroke or TIA & $8(23.5)$ & $43(24.0)$ & 0.97 & 0.41 to 2.31 & 0.9508 \\
\hline Arterial hypertension & $20(58.8)$ & $132(73.7)$ & 0.51 & 0.24 to 1.09 & 0.0777 \\
\hline Previous cardioversion & $4(11.8)$ & $4(2.2)$ & 5.83 & 1.38 to 24.72 & 0.0239 \\
\hline Digoxin treatment & $21(61.8)$ & $63(35.2)$ & 2.97 & 1.39 to 6.36 & 0.0037 \\
\hline
\end{tabular}

Differences were considered significant at $\mathrm{p}<0.05$. Other variables listed in table 1 did not differ between both groups.

${ }^{\star}$ Mean values and $t$ test. For other variables: values (\%) and $\chi^{2}$ test, with Yates' correction or Fisher's exact test when appropriate. 
Table 3 Comparison between patients with and without antithrombotic treatment (oral anticoagulant or antiplatelet drugs) at admission

\begin{tabular}{|c|c|c|c|c|c|}
\hline & \multicolumn{2}{|c|}{ Any antithrombotic } & \multirow[b]{2}{*}{ Odds ratio } & \multirow{2}{*}{$\begin{array}{l}95 \% \text { confidence } \\
\text { intervals }\end{array}$} & \multirow[b]{2}{*}{$p$ Value } \\
\hline & Yes $(n=99)$ & No $(n=114)$ & & & \\
\hline Age $(\text { years })^{\star}$ & 76.6 & 7871 & & & 0.0870 \\
\hline Atrial fibrillation previously known & $84(84.9)$ & $64(56.1)$ & 4.38 & 2.25 to 8.50 & 0.0001 \\
\hline Ischaemic stroke & $63(63.6)$ & $88(77.2)$ & 0.52 & 0.28 to 0.94 & 0.0298 \\
\hline Haemorrhagic stroke & $14(14.1)$ & $4(3.5)$ & 4.53 & 1.43 to 14.32 & 0.0054 \\
\hline Previous stroke or TIA & $28(28.3)$ & $23(20.2)$ & 1.56 & 0.83 to 2.94 & 0.1667 \\
\hline Arterial hypertension & $63(63.6)$ & $89(78.1)$ & 0.49 & 0.27 to 0.90 & 0.0201 \\
\hline Smoking & $5(5.1)$ & $19(16.7)$ & 0.27 & 0.10 to 0.74 & 0.0075 \\
\hline Past oral anticoagulation & $10(10.1)$ & $2(1.8)$ & 6.29 & 1.34 to 29.62 & 0.0084 \\
\hline Digoxin treatment & $47(47.5)$ & $37(32.5)$ & 1.88 & 1.08 to 3.29 & 0.0253 \\
\hline Antiarrhythmic treatment & $31(31.3)$ & $22(19.3)$ & 1.91 & 1.01 to 3.59 & 0.0431 \\
\hline
\end{tabular}

Differences were considered significant at $\mathrm{p}<0.05$. Other variables listed in table 1 did not differ between both groups.

${ }^{\star}$ Mean values and $t$ test. For other variables: values (\%) and $\chi^{2}$ test, with Yates' correction or Fisher's exact test when appropriate.

\section{Results}

Of 213 patients with atrial fibrillation who were admitted for stroke or TIA, $34(16.0 \%)$ were receiving anticoagulation treatment before stroke, and 99 (46.5\%) were receiving any antithrombotic drugs, including three patients who had both anticoagulation and antiplatelet treatment. One hundred and fourteen $(53.5 \%)$ were not receiving any antithrombotic treatment before their stroke. Of 34 patients who were receiving anticoagulant treatment, only six $(17.6 \%)$ were properly treated, while 15 $(44.1 \%)$ were undertreated, and three $(8.8 \%)$ were overtreated. In 10 patients, INR values were not determined because of obvious non-compliance, long delays between stroke onset and admission, and recent interruption of treatment for medical reasons (anaemia or surgery). On the other hand, at discharge, of 178 survivors, $154(86.5 \%)$ received anticoagulant or antiplatelet treatment.

Atrial fibrillation was previously known in $148(69.5 \%)$ patients and the mean delay between the onset of atrial fibrillation and stroke ranged from $0-22$ years. Of these 148

Table 4 Logistic regression analysis with oral anticoagulation (1 if present, 0 if absent) as dependent variable

\begin{tabular}{lrll}
\hline & \multicolumn{1}{l}{$S$} & \multicolumn{1}{l}{$S E$} & \multicolumn{1}{l}{$p$} \\
\hline Digoxin & 1.0366 & 0.4030 & 0.0101 \\
Arterial hypertension & -0.8906 & 0.4156 & 0.0321 \\
Mitral stenosis & 1.7183 & 0.8069 & 0.0332 \\
Cardioversion & 1.5885 & 0.7922 & 0.0450
\end{tabular}

Candidate variables were chosen as variables associated with current anticoagulation treatment at admission in the bivariate analysis, with a value of $p<0.25$, and consisting of clinical variables assessable before stroke onset: age (years), male sex, previously known atrial fibrillation, mitra stenosis, previous systemic emboli, depression, peripheral artery disease, arterial hypertension, hyperlipidaemia, other high risk cardiopathies, cardioversion, digoxin, $\beta$ blockers, calcium channel blockers, and centre $=$ Paris (all quoted $0=$ no and $1=$ yes). Colinearity between variables (defined as $r>0.6$ ) was excluded. Overall prediction of the model $84.98 \%$.

$\beta$, regression coefficient; $S E$, standard error of the mean; constant $=-1.7602$.

Table 5 Logistic regression analysis with any antithrombotic drug at admission (1 if present, 0 if absent) as dependent variable

\begin{tabular}{lrll}
\hline & $\beta$ & $S E$ & $p$ \\
\hline Known atrial fibrillation & 1.6735 & 0.3742 & 0.0000 \\
Age & -0.0411 & 0.0172 & 0.0169 \\
Smoking & -1.3355 & 0.5726 & 0.0197 \\
Arterial hypertension & -0.6934 & 0.3446 & 0.0442 \\
\hline
\end{tabular}

Candidate variables were chosen as variables associated with current anticoagulation at admission in the bivariate analysis, with a value of $\mathrm{p}<0.25$, and consisting of clinical variables assessable before stroke onset: age (years), previously known atrial fibrillation, previous stroke or TIA, previous systemic emboli, non-life threatening bleedings, arterial hypertension, hyperlipidaemia, smoking, past oral anticoagulation, cardioversion, digoxin, diuretics (all quoted $0=$ no and $1=$ yes). Colinearity between variables (defined as $r>0.6$ ) was excluded. Overall prediction of the model: $70.42 \%$.

Constant $=2.4254$. patients, $118(79.7 \%)$ were not receiving oral anticoagulation treatment and $64(43.2 \%)$ did not receive any antithrombotic drug. Of 137 patients eligible for oral anticoagulation (as previously defined), $108(78.8 \%)$ were not receiving such treatment; of 142 patients eligible for any antithrombotic treatment, 62 $(43.7 \%)$ were not receiving oral anticoagulation or antiplatelet treatment.

In the bivariate analysis comparing variables between patients who were receiving oral anticoagulation treatment before stroke and patients who were not, those receiving such treatment before stroke were more likely to have haemorrhagic stroke, previously known atrial fibrillation, mitral stenosis, to have had cardioversion, and to be currently receiving digoxin treatment; they were also less likely to have ischaemic stroke, but they did not differ in regard to history of previous stroke or TIA and arterial hypertension (table 2). Patients receiving any antithrombotic treatment before stroke were more likely to have haemorrhagic stroke, previously known atrial fibrillation, to have been receiving (interrupted) anticoagulants, and to be currently receiving digoxin or any other antiarrhythmic treatment; they were less likely to be admitted for ischaemic stroke, to have arterial hypertension, and to be smokers, but they did not differ in regard to history of previous stroke or TIA (table 3).

The logistic regression analysis assuming anticoagulation treatment before stroke as dependent variable found digoxin treatment, absence of arterial hypertension, mitral stenosis, and history of cardioversion as independent variables (table 4). The logistic regression analysis assuming any antithrombotic treatment before stroke as dependent variable found known atrial fibrillation, lower age, being a non-smoker, and absence of arterial hypertension as independent variables (table 5).

\section{Discussion}

Our study has shown that in consecutive patients with stroke and atrial fibrillation, only one sixth were receiving anticoagulant treatment before their stroke and less than half received either anticoagulant or antiplatelet treatment.

Our study was hospital based and does not provide any information about patients with atrial fibrillation who did not have a stroke 
within the study period, and about patients with stroke leading to death before hospitalisation. Conversely we cannot exclude that some patients with TIA were not recognised, not admitted to a stroke unit, or admitted to cardiological wards. However, despite any recruitment bias, this study was conducted in patients we should focus on - that is, those who have atrial fibrillation and have experienced a cerebrovascular event. Their major demographic characteristics did not differ from those of stroke patients included in community based registries. ${ }^{27}$

Our study was observational and did not try to determine why patients were not treated according to guidelines. The aim was to determine the proportion of patients who were not treated according to the guidelines, even if the reasons were good-for example, having a non-recognised atrial fibrillation. This information is important because if a reason for undertreatment is underrecognition of atrial fibrillation, information campaigns directed at recognising atrial fibrillation in the community may be necessary. This is why we also included patients whose atrial fibrillation was not known before stroke onset, and who were not candidates for antithrombotic treatment.

We cannot exclude the possibility that patients who received neither anticoagulation nor antiplatelet treatment may have had contraindications. However, this seems unlikely for two reasons: the rate of non-prescription of antithrombotic treatment seems to be too high to be explained just by contraindications; and at discharge 154 patients were receiving anticoagulants or antiplatelet treatment, leading to an $86.5 \%$ rate of prescription in survivors. The $13.5 \%$ rate of non-prescription, which included transient or permanent contraindications such as very large infarcts, haemorrhagic infarcts, or primary intracerebral haemorrhages, is probably much higher than it should have been theoretically before stroke.

Even in patients who received oral anticoagulation treatment, the INR values were not always within the target range. Because of the study design, we do not know the interval between the last dose and the time of blood sampling for INR, but in some patients the delay between stroke onset and admission may have skewed the results towards an overrepresentation of patients with low INR values.

Four variables were associated with prescription of oral anticoagulation: digoxin treatment; absence of arterial hypertension; presence of mitral stenosis; and history of cardioversion. The relation with the lack of arterial hypertension may reflect a reluctance on the part of practitioners to give oral anticoagulation treatment to hypertensive patients, or at least to patients with non-stabilised arterial hypertension. This is part of many recommendations, ${ }^{13}$ but the best approach is probably to lower blood pressure after the acute stage, and provide oral anticoagulation treatment for patients with atrial fibrillation, because arterial hypertension is associated with an increased risk of ischaemic stroke in these patients. ${ }^{10}$ Although there is, to our knowledge, no randomised trial proving that oral anticoagulation treatment is safe and effective in reducing the risk of stroke in patients with atrial fibrillation and mitral stenosis, it has been recommended in these patients for many years in light of the findings of the Framingham study which showed the high risk of stroke in such patients. ${ }^{28}$ Therefore, it is not surprising that mitral stenosis is associated with a higher rate of oral anticoagulation treatment in patients with atrial fibrillation.

Another explanation for the higher rate of anticoagulation treatment in patients with mitral stenosis may be that these patients are more likely to be followed by a cardiologist, as well as patients with digoxin treatment or cardioversion. Cardiologists may be less reluctant to use oral anticoagulation treatment than GPs, but this hypothesis has to be evaluated. The type of atrial fibrillation, either paroxysmal, persistent or permanent, was not taken into account in our study as far as most trials. ${ }^{7}$ It is not possible to determine if the type of atrial fibrillation influences the prescription of oral anticoagulant treatment, but the pooled data ${ }^{10}$ suggest that patients with paroxysmal NVAF have the same risk of stroke as patients with chronic NVAF. History of stroke or TIA was surprisingly not associated with a higher rate of anticoagulation treatment, despite the results of the European atrial fibrillation trial. ${ }^{7}$ As reported for myocardial infarction, ${ }^{29}$ it seems that such an event does not modify therapeutic attitudes concerning vascular risk factors.

Recommendations for the use of oral anticoagulation treatment in patients with NVAF state no upper age limit. ${ }^{30}$ In clinical studies, the mean age of patients was lower than 70 years, and only $20 \%$ were over 75 years. Many physicians may remain uncertain about the safety of oral anticoagulation treatment in the elderly and argue that drug trials were conducted in younger patients. However, the SPAF III study, by determining groups of high risk patients, has clearly shown the benefit of anticoagulation treatment in patients older than 75 years, especially women. ${ }^{11}$ Despite this result, a recent study has shown that anticoagulants are underused in elderly patients with atrial fibrillation, particularly in women older than 75 years. $^{31}$ The results of the stroke prevention in reversible ischaemia trial $(\text { SPIRIT })^{32}$ may probably lead to an increased reluctance on the part of physicians to use anticoagulants in the elderly. However, it should be borne in mind that SPIRIT was conducted in patients with non-cardioembolic strokes and that the level of anticoagulation treatment was much higher.

Four variables were associated with prescription of any antithrombotic treatment: known atrial fibrillation; lower age; being a nonsmoker; and absence of arterial hypertension. The relation with lower age and the lack of arterial hypertension may also reflect a reluctance on the part of practitioners to give hypertensive or elderly patients antithrombotic treatments. The prescription of antithrombotic treatment was influenced by the knowledge of atrial fibrillation, although prescription of oral 
anticoagulation was not. However, 64 of 114 patients $(56.1 \%)$ without any antithrombotic treatment had a known atrial fibrillation. The statistical relation between being a non-smoker and receiving any antithrombotic treatment remains unexplained.

\section{Conclusion}

The SAFE I study has shown that more than half of those patients admitted for acute stroke or TIA who had atrial fibrillation were not receiving any antithrombotic treatment before their stroke. Advanced age and arterial hypertension were associated with a lower rate of prescription. The reasons for such undertreatment remain to be determined.

To address this question, a large prospective multicentre and multinational study, including referral and non-referral, university and nonuniversity hospitals, is required. This will be the aim of SAFE II. SAFE II may include other important social or medical variables which should be taken into account when prescribing anticoagulation treatment, such as the patient's level of education, being examined by a cardiologist over the previous 12 months, the number of medical contacts over the previous 12 months, having cognitive decline, living alone, or living in a rural, suburban or urban area.

One of the most likely reasons for undertreatment may be the failure of the conclusions from clinical trials to translate into change in clinical practice. If this is true, information campaigns will be required. In terms of public health, they may be as useful as new trials. In this context, GPs and cardiologists have a crucial role in detecting atrial fibrillation and instituting adequate treatment, because they are more likely to see these patients before they have a stroke.

This trial was supported by the Association pour la recherche et l'enseignement en pathologie neurovasculaire (APREPAN) and Equipe d'accueil EA 2691 (Ministère
de la Recherche et de la Technologie).

\section{Appendix}

LIST OF PARTICIPANTS

Writing committee

Dominique Deplanque, Francesco Corea, Caroline Arquizan, Lucilla Parnetti, Jean-Louis Mas, Virgilio Gallai, Didier Leys.

Data management

Dominique Deplanque, Francesco Corea, Caroline Arquizan.

Statistical analysis

Dominique Deplanque, Didier Leys.

Investigators

University of Perugia, Via Enrico dal Pozzo, I-06126

Perugia, Italy: Lucilla Parnetti, Francesco Corea, Gianluca Marziani, Raul Panico, Virgilio Gallai (Institute of Neurology)

Sainte-Anne Hospital, 1 rue Cabanis F-75674 Paris, France: Caroline Arquizan, Jean-Louis Mas (department of neurology); Laure Cabanes (department of cardiology).

University of Lille, Roger Salengro Hospital, F-59037 Lille, France: Christine Daems, Dominique Deplanque, Olivier Godefroy, Djelloul Guerouaou,
Hilde Hénon, Didier Leys, Christian Lucas (department of neurology); Xavier Leclerc, Jean-Pierre Pruvo (department of neuroradiology); Ghislaine Deklunder, Corinne Gautier (department of ultrasonology); Salem Kacet, Dominique Lacroix (department of cardiology)

1 Wolf PA, D'Agostino RB, Belanger AJ, et al. Probability of stroke: a risk profile from the Framingham study. Stroke 1991;22:312-18.

2 Wolf PA, Dawber TR, Thomas HE Jr, et al. Epidemiological assessment of chronic atrial fibrillation and the risk of assessment of chronic atrial fibrillation and the risk of
stroke: the Framingham study. Neurology 1978;28:973-7.

3 Jørgensen HS, Nakayama H, Reith J, et al. Acute stroke with atrial fibrillation: the Copenhagen stroke study. Stroke 1996;10:1765-9.

4 Lin HJ, Wolf PA, Kelly-Hayes M, et al. Stroke severity in atrial fibrillation: the Framingham study. Stroke 1996;27: 1760-4.

5 Stroke Prevention in Atrial Fibrillation Investigators. Predictors of thromboembolism in atrial fibrillation: part I. Clinical features of patients at risk. Ann Intern Med 1992;116:1-5.

6 Stroke Prevention in Atrial Fibrillation Investigators: predictors of thromboembolism in atrial fibrillation: part II. Echocardiographic features of patients at risk. Ann Intern Med 1992;116:6-12.

7 European Atrial Fibrillation Trial Study Group. European atrial fibrillation trial: secondary prevention in nontrial fibrillation trial: secondary prevention in nonrheumatic atrial fibrillation after transient isch

8 Hart RG, Sherman DG, Easton JD, et al. Prevention of stroke in patients with nonvalvular atrial fibrillation. Neurology 1998;51:674-81.

9 Sharif MN, Wyse DG. Atrial fibrillation: overview of therapeutic trials. Can f Cardiol 1998;14:1241-54.

10 Atrial Fibrillation Investigators. Risk factors for stroke and efficacy of antithrombotic therapy in atrial fibrillation: analysis of pooled data from five randomized controlled trials. Arch Intern Med 1994;154:1449-57.

11 SPAF III Writing Committee for the Stroke Prevention in Atrial Fibrillation Investigators. Patients with nonvalvular atrial fibrillation at low risk of stroke during treatment with aspirin: stroke prevention in atrial fibrillation III study. aspirin: stroke prevention

12 McCrory DC, Matchar DB, Samsa G, et al. Physician attitudes about anticoagulation for nonvalvular atrial fibrillation in the elderly. Arch Intern Med 1995;155:277-81.

13 Kutnner M, Nixon G, Silverstone F. Physicians attitudes toward oral anticoagulant and antiplatelet agents for stroke prevention in elderly patients with atrial fibrillation. Arch ntern Med 1991;151:1950-3.

14 Brodsky MA, Chun JG, Podrid PJ, et al. Regional attitudes of generalists, specialists and subspecialists about management of atrial fibrillation. Arch Intern Med 1996;156:255362

15 Brass LM, Krumholz HM, Scinto JM, et al. Warfarin use among patients with atrial fibrillation. Stroke 1997;28 2382-9

16 Beyth RJ, Antani MR, Covinsky KE, et al. Why isn't warfarin prescribed to patients with nonrheumatic atrial fibrillation? F Gen Intern Med 1996;11:721-8.

17 Antani MR, Beyth RJ, Covinsky KE, et al. Failure to prescribe warfarin to patients with nonrheumatic atrial prescribe warfarin to patients with nonrheu

18 Enis J. Stroke prevention in patients with non-valvular atrial fibrillation: a current community perspective. F Clin Neurofibrillation: a current com
science 1994;4:320-5.

19 Bath PM, Prasad A, Brown MM, et al. Survey of the use of anticoagulation in patients with atrial fibrillation. $B M \mathcal{F}$ 1993;307:1045

20 Gariballa SE, Robinson TG, Parker SG, et al. A propective study of primary and secondary risk factor management in stroke patients. F R Coll Physicians Lond 1995;29:485-7.

$21 \mathrm{Lip}$ GYH, Zarifis J, Watson RDS, et al. Physician variation in the management of patients with atrial fibrillation. Heart 1996;75:200-5.

22 Gottlieb LK, Salem-Schatz S. Anticoagulation in atrial fibrillation: does efficacy in clinical trials translate into effectiveness in practice? Arch Intern Med 1994;154:1945-53.

23 Masuhr F, Busch M, Einhäupl KM. Differences in medical and surgical therapy for stroke prevention between leading experts in North

24 Kalra L, Perez I, Melbourn A. Stroke risk management: changes in mainstream practice. Stroke 1998;29:53-7.

25 Adams HP Jr, Bendixen BH, Kappelle LJ, et al and the TOAST Investigators. Classification of subtypes of acute ischemic stroke: definitions for use in a multicenter clinical trial. Stroke 1993;24:35-41.

26 Bendel RB, Afifi AA. Comparison of stopping rules in forward regression. 7 Am Stat Assoc 1977;72:46-53.

27 Giroud M, Milan C, Beuriat P, et al. Incidence and survival rates during a two-year period of intracerebral and subarachnoid haemorrhages, cortical infarcts, lacunes and transient ischemic attacks: the stroke registry of Dijon, 1985-1989. Int f Epidemiol 1991;20:892-9.

28 Kannel WB, Abbott RD, Savage DD, et al. Epidemiologic features of chronic atrial fibrillation: the Framingham features of chronic atrial fibrillation:
study. $N$ Engl f Med 1982;306:1018-22. 
29 EUROASPIRE Study Group. European action on secondary prevention through intervention to reduce events. A European Society of Cardiology survey of secondary prevention of coronary he

30 Laupacis A, Albers G, Dalen J, et al. Antithrombotic therapy in atrial fibrillation. Chest 1995;108(suppl):352-9.
31 Sudlow M, Thomson R, Thwaites B, et al. Prevalence of atrial fibrillation and eligibility for anticoagulants in the atrial fibrillation and eligibility for an

32 Stroke Prevention in Reversible Ischemia Trial (SPIRIT) Study Group. A randomized trial of anticoagulants versus aspirin after cerebral ischemia of presumed arterial origin. Ann Neurol 1997;42:857-65.

\section{IMAGES IN CARDIOLOGY}

\section{The Vineberg procedure}

This 66 year old white man had had an inferior myocardial infarction and coronary artery bypass surgery (CABG) in 1980. He underwent five vessel CABG in 1980 for refractory angina with severe three vessel coronary artery disease. $\mathrm{He}$ received several saphenous vein grafts. The left internal mammary artery (LIMA) was sutured directly into the posterior left ventricular wall using the Vineberg technique.

$\mathrm{He}$ had been angina free since surgery in 1980; however, a decrease in exercise tolerance prompted exercise echocardiographic stress testing in 1999. The resting echocardiogram showed mild inferior hypokinesis. He exercised for 6 minutes 47 seconds on the standard Bruce protocol, stopping for fatigue. There were no ECG changes suggestive of ischaemia. Echocardiography following exercise showed inferior dyskinesis and anteroseptal hypokinesis with normal function of the posterolateral wall.

Coronary angiography demonstrated a $60 \%$ distal left main stenosis and occlusions of the proximal left anterior descending (LAD), left circumflex, and right coronary arteries. The saphenous vein graft to the obtuse marginal

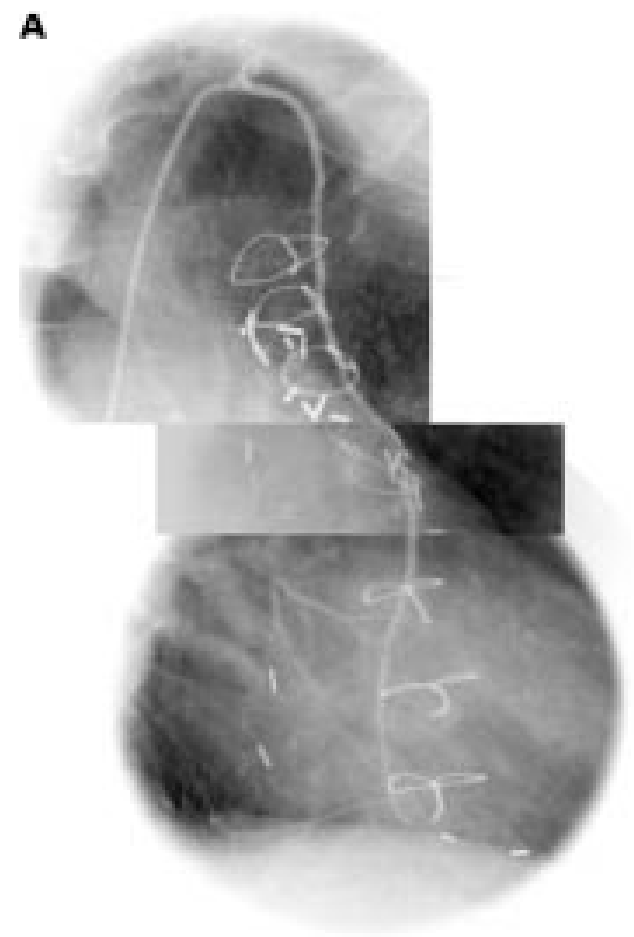

branch of the circumflex was occluded. The saphenous vein grafts to the LAD, diagonal, and posterior descending branch of the right coronary artery (PDA) were patent. There was high grade disease of the saphenous vein graft to the PDA. The LIMA to the myocardium was patent (A). A magnified view during LIMA angiography (B) showed collateral filling of the obtuse marginals (curved arrow) and PDA (straight arrow).

Promulgated by the Canadian surgeon Arthur Vineberg, implantation of the LIMA directly into the myocardial wall was performed in the 1960s. With the introduction of LIMA to coronary artery anastomosis toward the end of the decade, the Vineberg procedure was largely abandoned. This case demonstrates that 19 years following the Vineberg procedure, this patient still had a patent LIMA graft providing brisk collateral filling of the obtuse marginal and PDA. This graft is performing well, providing adequate blood flow to the posterolateral wall. The patient is currently feeling well continuing his antianginal medical regimen.

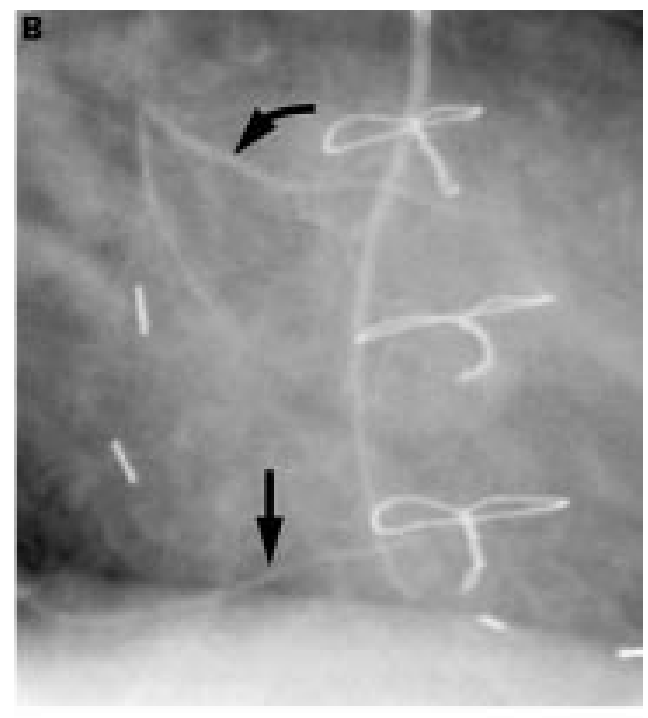

A D MICHAELS T M CHOU 